\title{
Middle-down electron capture dissociation and electron transfer dissociation for histone analysis
}

\author{
Annie Moradian ${ }^{1}$, Catarina Franco ${ }^{1,2}$, Michael J. Sweredoski ${ }^{1}$ and Sonja Hess ${ }^{1 *}$ (D
}

\begin{abstract}
The post-translational modifications (PTMs) of histones play a major role in activating or silencing gene transcription. To gain better understanding of the interplay between the PTMs that occur on histones, they are extensively studied using mass spectrometry techniques. Due to the abundance of lysines and arginines, the typical trypsin digestion has been found less favorable and GluC-digests have been explored as an alternative to yield larger peptides amenable to middle-down approaches. In addition, the use of weak cation exchange hydrophilic interaction liquid chromatography (WCX-HILIC) and the use of electron-based fragmentation techniques were found to be advantageous for the in-depth characterization of histone variants containing multiple PTMs.

As a test model, we used histones from MEL (murine erythroleukemia) cells treated with butyric acid or DMSO. After acid extraction, histone pellets were dried and fractionated using a reversed-phase C3 column. For middle-down analysis, selected histone fractions were digested using GluC. The digested samples were separated on a WCX-HILIC capillary column packed in-house with PolyCAT A resin, coupled to a linear trap quadrupole Fourier transformation ion cyclotron resonance (LTQFT-ICR) instrument. Raw data was acquired on the LTQFT-ICR using electron capture dissociation (ECD). After deconvolution of the raw data, we generated heatmaps to illustrate differential maps between differentially treated histone samples. We also explored the innovative use of Skyline to quantify histone tails. In addition, we report some preliminary data using a synthetic histone peptide acquired on an Orbitrap Fusion using electron transfer dissociation (ETD). Both, ECD and ETD methods are capable of comprehensively analyzing complex histone variations not accessible with conventional techniques.
\end{abstract}

Keywords: Histone; Post-translational modification; Middle-down proteomics; Electron capture dissociation; Electron transfer dissociation; Data-dependent analysis; Data-independent analysis

\section{Review}

The post-translational modifications (PTMs) of histones play a major role in activating or silencing gene transcription. Acetylation and methylation are the two most common PTMs on histones. Acetylation occurs on lysines and is transferred by histone acetylases and removed by histone deacetylases. Histone methyl transferases and demethylases regulate the mono-, di- and tri-methylation of lysines and arginines. Acetylation and methylation have opposite effects: methylation stabilizes the charge of the side chain amine and amidine, respectively, while acetylation neutralizes the

\footnotetext{
*Correspondence: shess@caltech.edu

${ }^{1}$ Proteome Exploration Laboratory, Beckman Institute, California Institute of Technology, BI 211, MC 139-74, Pasadena, CA 91125, USA

Full list of author information is available at the end of the article
}

amine of the lysine by forming an amide bond (Fig. 1a). A general model assumes that the positively charged lysines interact closely with the negatively charged DNA, leading to a closed chromatin formation that is associated with transcriptional repression. In contrast, acetylated and thus neutral lysines cannot interact closely with DNA, leading to an open chromatin structure, which is commonly associated with transcriptional activation (Fig. 1b) (Garcia et al. 2007).

Mass spectrometry (MS) and particularly middle-down approaches are playing an increasingly important role in characterizing PTMs on histones (Cannon et al. 2010; Garcia 2010; Kalli et al. 2013; Wu et al. 2005; Moradian et al. 2014). In contrast to a typical bottom-up approach, where proteins are digested with trypsin to generate peptides generally smaller than $2500 \mathrm{Da}$, middle-down 
A

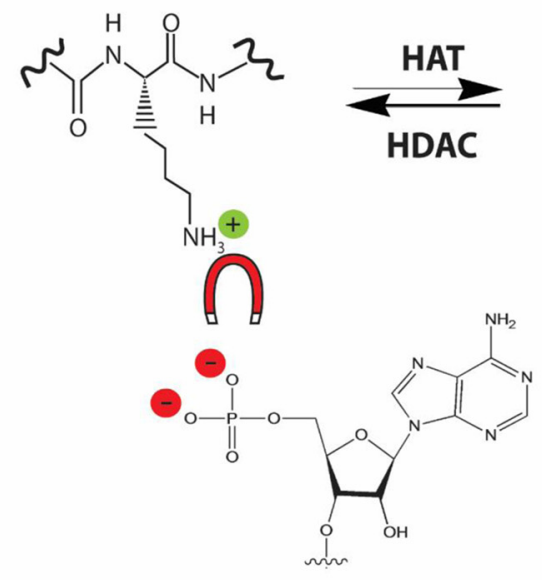<smiles>[Z]NC(=O)[C@H](CCCCNC(C)=O)NC(C)=O</smiles>

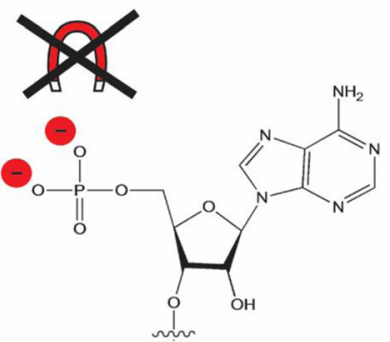

B


Transcriptional activation

Fig. 1 a Histone lysine side chains can be acetylated via histone acetylases and deacetylated via histone deacetylases. The acetylation creates a newly formed amide bond that cannot be protonated. b Thus, acetylated histones cannot interact closely with negatively charged DNA, opening up chromatins to their active form

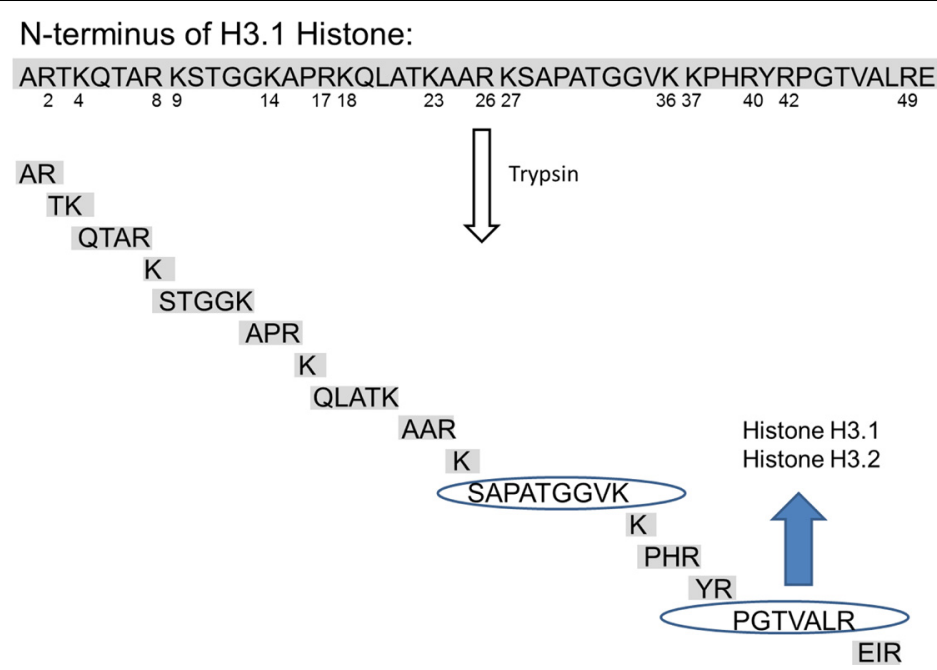

Fig. 2 Example of a theoretical tryptic digestion of the N-terminus of H3.1 histone leading to peptides generally smaller than six amino acids that would not be amenable to LC-MS/MS in a typical bottom-up approach. Only two peptides (in blue circles) would be detectable. In addition, peptide PGTVALR is shared between histone $\mathrm{H} 3.1$ and histone H3.2, which makes it impossible to infer from which protein it originally came from. Middle-down approaches circumvent these problems by looking at larger peptides (in the case of histone H3.1 with 50 amino acids) 

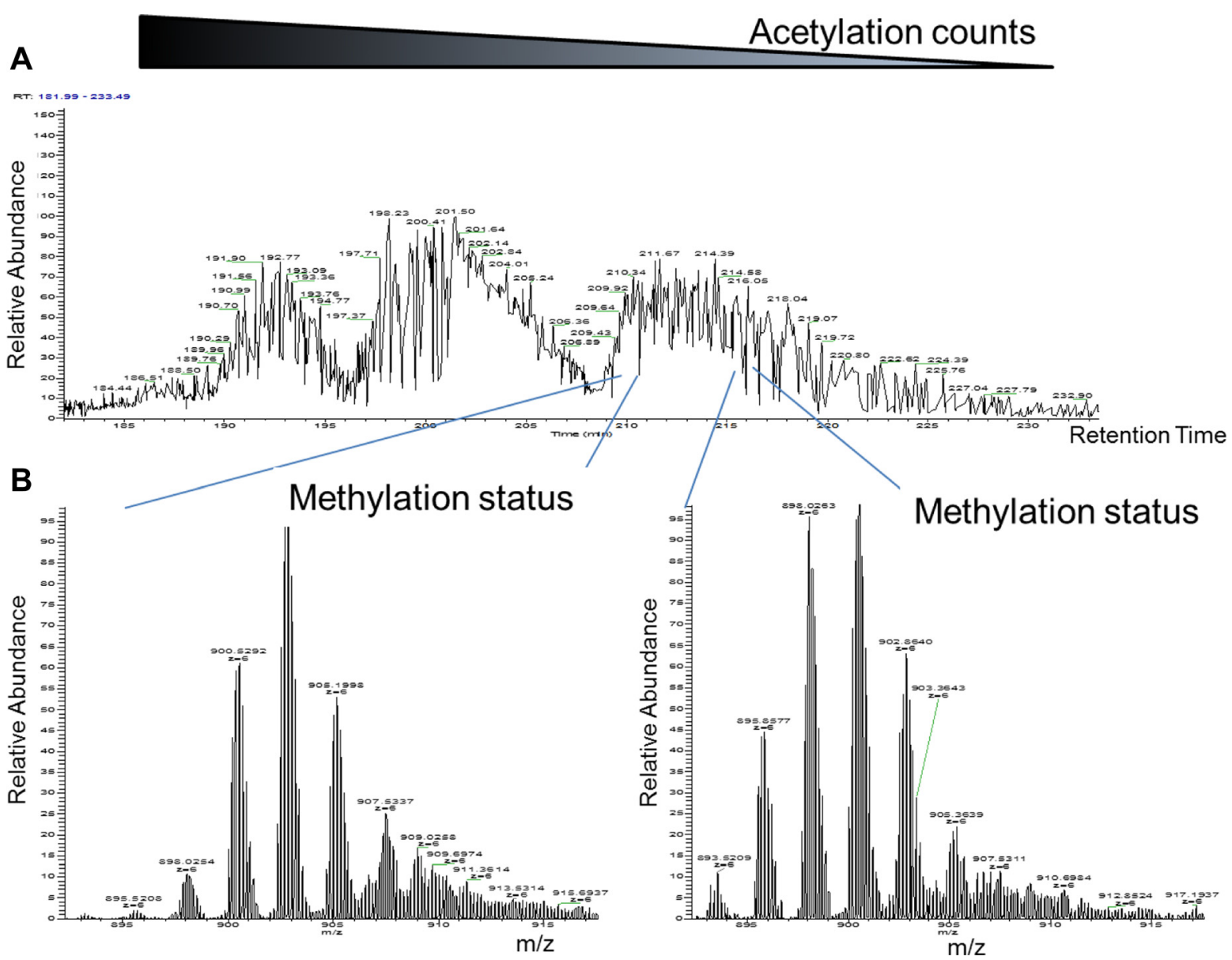

Fig. 3 WCX-HILIC separation of histone tails enables the separation based on acetylation (a) and methylation (b) status


Fig. 4 By fragmenting the peptide bonds, collision-induced dissociation (CID) produces b- and y-ions of unequal intensity, while electron capture/transfer dissociation (EC/TD) fragments between the alpha carbon and the amine adjacent to the peptide bonds, thus producing c- and z-ions of approximately the same intensity, aiding both the identification of PTMs and the quantification of the peptides. A comparison for the synthetic H3 histone N-terminal fragment ARTKQTARKSTGGKAPRKQLATKAARKSAPATGGVKKPHRYRPGTVALRE is shown with CID (a) and ETD (b) 
approaches use enzymes that yield larger peptides (circa $3000-10,000 \mathrm{Da}$ ). This is particularly useful for histones, where lysines and arginines are abundant in the N-termini. As shown in Fig. 2, trypsin would typically generate histone peptides too small to be reliably detected by MS. In addition, many histone proteoforms share sequence homology (e.g., peptide PGTVALR is shared by histone H3.1 and H3.2). Thus, even if a peptide is detected and characterized, it would be impossible to infer from which proteoform the PTMs originated from. Both AspN and GluC have been successfully used to generate middle-down peptides from histones (Kalli et al. 2013; Bonenfant et al. 2006; da Cunha et al. 2006; Guedes et al. 2011; Jung et al.
2013; Kawasaki et al. 2003; Lu et al. 2009; Kalli and Hess 2012; Phanstiel et al. 2008). The use of weak cation exchange hydrophilic interaction liquid chromatography (WCX-HILIC) enabled the separation of histones based on their charge (acetylation status) and hydrophilic interactions (methylation status) (Young et al. 2009; Young et al. 2010). Fig. 3a shows the resulting broad peaks presenting 5, 4, 3, etc. acetylations. While the peaks may appear relatively broad in comparison to C18 reversed-phase chromatography, WCX-HILIC affords a secondary dimension of separation based on the methylation status (Fig. 3b), separating multiple methylated isoforms that contain the same number of acetylations.
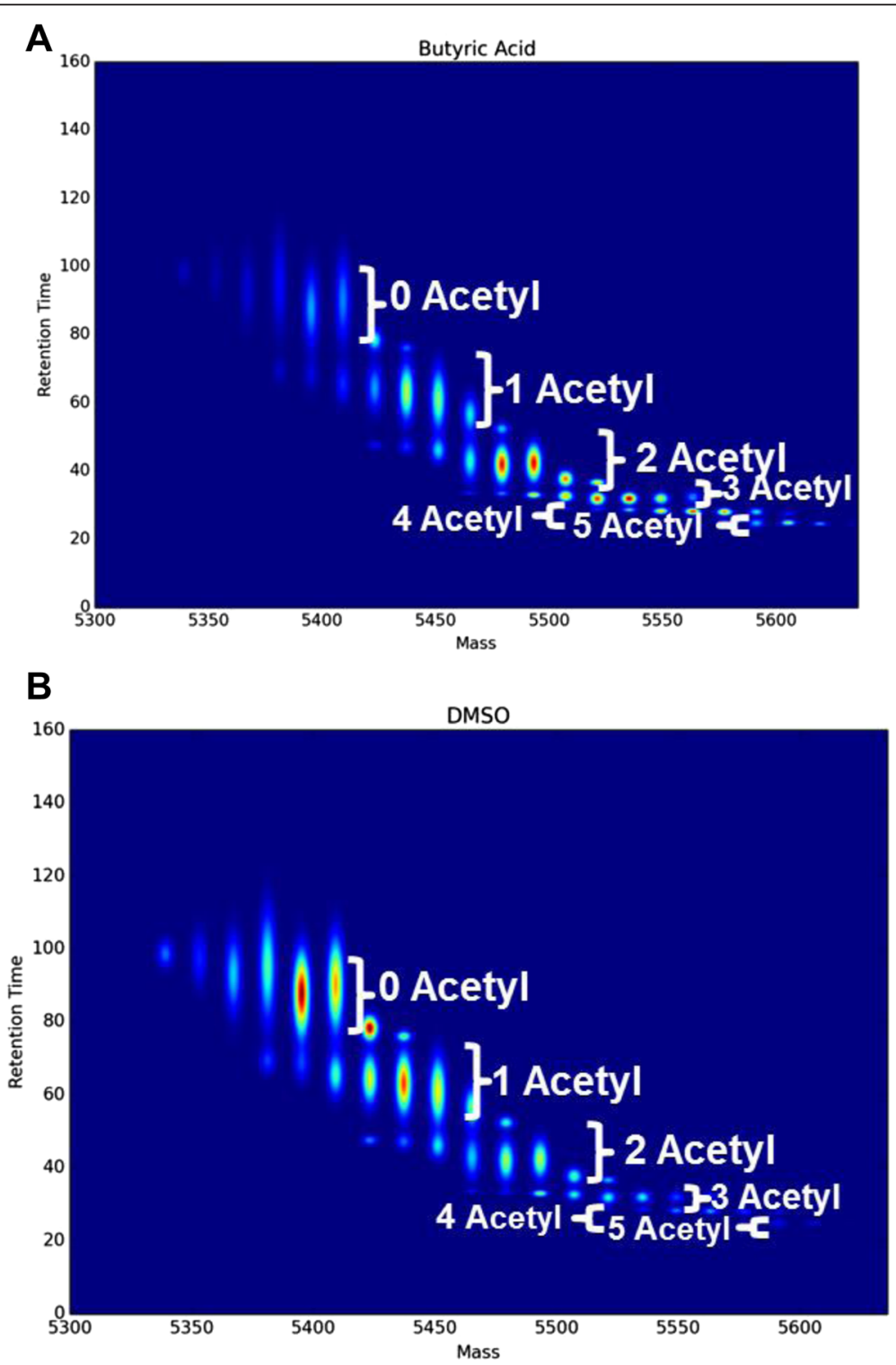

Fig. 5 Heat map of the MS1 scans vs. deconvoluted mass of the GluC-digested N-terminus of histone H3 spectra treated with (a) butyric acid and (b) DMSO after WCX-HILIC separation. The histone deacetylase inhibitor butyric acid-treated H3 histones showed the most intense ions with 2 and 3 acetylations, while the DMSO-treated $\mathrm{H} 3$ histones showed the most intense ions with 0 and 1 acetylations 


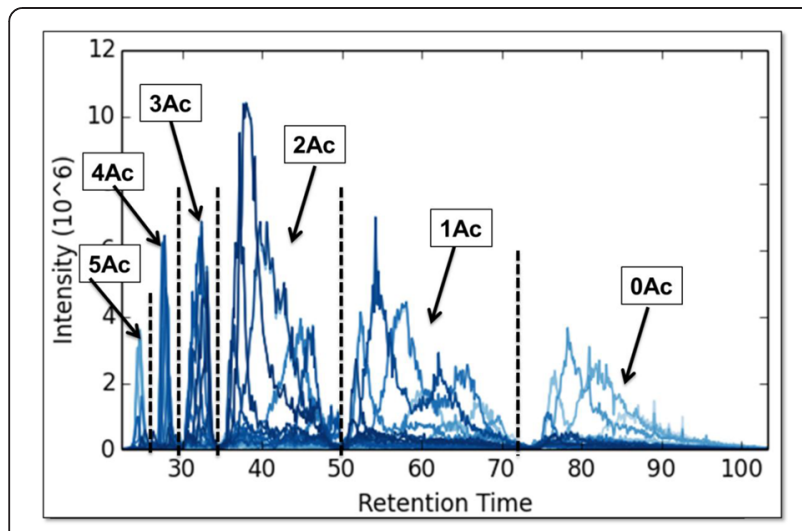

Fig. 6 Using Skyline to quantify changes of acetylation status in $\mathrm{H} 3$ histones after importing all theoretical +10 ions for all possible histone H3 tails with $0-5$ acetylations and $0-7$ methylations

Young et al. introduced a $\mathrm{pH}$ gradient using WCX-HILIC column to allow direct online coupling of WCX-HILIC with MS (Young et al. 2009; Young et al. 2010). A recent further improvement using a 2D column setup with a C18 precolumn for sample loading has been introduced by Jung et al. (2013). The use of a C18 precolumn allowed Jung et al. to dissolve samples in aqueous solutions rather than acetonitrile solutions that were necessary for the WCX-HILIC approach. This improved reproducibility, since the acetonitrile solutions evaporated too quickly during analysis.

As a mild non-ergodic fragmentation technique that preserves PTMs, electron transfer dissociation (ETD) and electron capture dissociation (ECD) have been proven beneficial for the detection of histone modifications. In addition, fragmentation during electron-based dissociation yields more equally intense fragment ions, when compared to collision-induced dissociation (CID) (Fig. 4). An additional benefit is the fact that ratios of fragment ion intensities in electron-based fragmentation techniques can be used for proteoform quantitation (Pesavento et al. 2006).

In our own lab, we use histones from MEL cells as a test model. When treated with the histone deacetylase inhibitor butyric acid or DMSO for 4 days, MEL cells differentiate and produce embryonic or adult hemoglobin, respectively. After acid extraction (Shechter et al. 2007), histone pellets were dried and fractionated using a reversed-phase C3 column. For middle-down analysis, selected histone fractions were digested using GluC. The digested samples were separated on a WCX-HILIC capillary column packed in-house with PolyCAT A resin, coupled to a linear tap quadrupole Fourier transformation ion cyclotron resonance (LTQFT-ICR). After deconvolution of the raw data using YADA (Carvalho et al. 2009), we generated idealized heatmaps to illustrate differences between butyric acid and DMSO-treated histone samples (Fig. 5). As expected, the deacetylase inhibitor butyric acid inhibited deacetylation of histones. In general, the most intense ions were represented by $2-4$ acetylations (Fig. $5 \mathrm{a}$ ). In contrast, the DMSO-treated histones showed more intense ions for 0 and 1 acetylations as shown in Fig. 5 b.

To make efficient use of the precursor ion intensities for quantitative purposes, we also explored the innovative use of Skyline to quantify histone tails. Since the +10 fragment ions were the most intense ions in the MS spectra, we imported all theoretical +10 ions for all possible histone H3.2 tails with $0-5$ acetylations and $0-7$ methylations in Skyline (Schilling et al. 2012; MacLean et al. 2010). The resulting total ion chromatogram (XIC) is shown in Fig. 6 , quantifying all acetylation statuses identified in the WCXHILIC LTQFT ECD analyses.

In addition to ECD analyses on the LTQFT, we have started to explore suitable instrument parameters for ETD on an Orbitrap Fusion. To do so, preliminary data of the synthetic $\mathrm{H} 3$ histone $\mathrm{N}$-terminal fragment

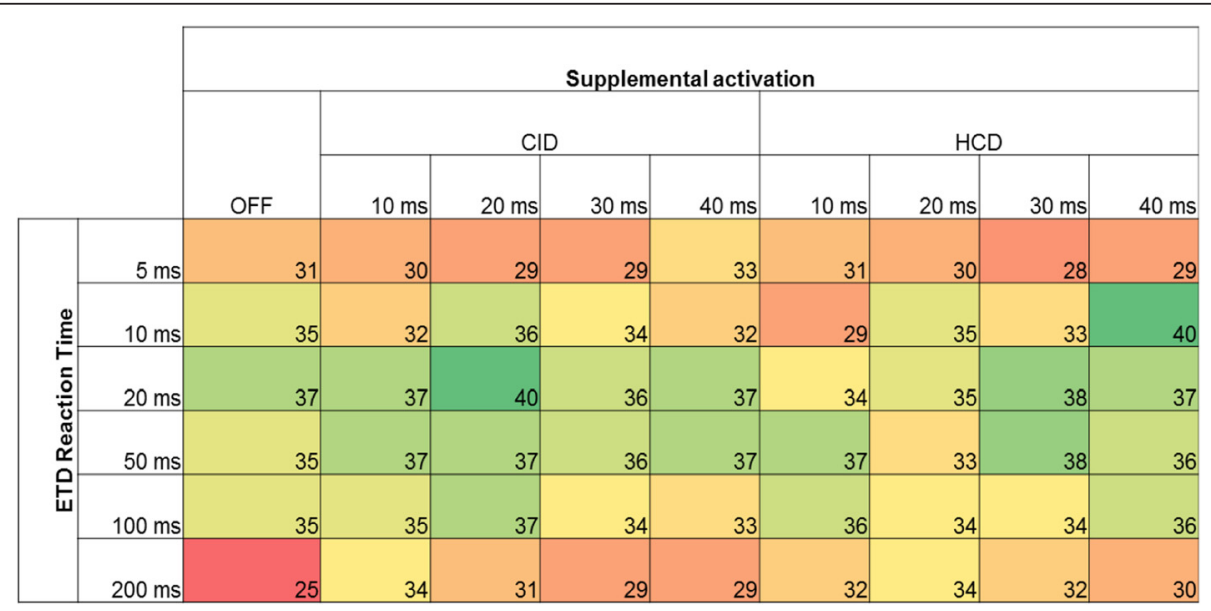

Fig. 7 Optimization of fusion ETD acquisition parameters. Number of peptide cleavages observed for synthetic H3 fragment using different ETD reaction times vs. supplemental activation with CID or HCD 
ARTKQTARKSTGGKAPRKQLATKAARKSAPATGGVK KPHRYRPGTVALRE were acquired on an Orbitrap Fusion instrument.

Since the $5 \mathrm{kDa}$ peptide is more like a small protein than a peptide, we tested initially whether standard pressure settings or intact protein settings should be used for fragmentation. In all cases, the standard pressure settings showed more cleavages than the protein pressure settings. Next, we needed to convert the raw files into $m z x m l$ files to be further converted to MGF (Mascot Generic Format) files for database searching. For the raw file to $m z x \mathrm{xml}$ file conversion, we tested MSConvert (French et al. 2014), and for the $m z x m l$ file to MGF conversion, we used MS-Deconv (Liu et al. 2010). The combination of MSConvert and MS-Deconv performed similarly to ReAdW and MS-Deconv for the multiply charged ions as they are observed in middle-down approaches. As shown in Fig. 7, we found that short reaction times of $10 \mathrm{~ms}$ together with $40 \%$ supplemental higher energy collision dissociation (HCD) activation gave better results than longer reaction times and lower or higher activation energies. Similarly, 20-ms ETD reaction times together with 20-ms CID supplemental activation resulted in more detectable cleavages (Fig. 7). When we compared the different charge states $(+7,+8$, $+9,+10)$, we found that the most intense +10 ion generally gave more cleavage product ions.

\section{Conclusions}

Taken together, the use of WCX-HILIC with middledown proteomics is becoming an increasingly popular method for histone characterization. Our preliminary data furthermore show that both the LTQFT with ECD acquisition and the Orbitrap Fusion with ETD acquisition are capable of identifying and quantifying histone PTMs. We are predicting that the Orbitrap Fusion will play an important role in the comprehensive and reproducible analysis of histones. Fully automated bioinformatics pipelines as they exist for bottom-up proteomics are not yet available for middle-down proteomics and will require substantial development. Once in place, they will likely trigger another significant advancement in histone modification analysis.

\section{Abbreviations \\ ECD: Electron capture dissociation; ETD: Electron transfer dissociation; HCD: Higher energy collision dissociation; FT-ICR: Fourier transformation ion cyclotron resonance; HDAC: Histone deacetylase; WCX-HILIC: Weak cation exchange hydrophilic interaction liquid chromatography.}

\section{Competing interests}

The authors declare that they have no competing interests.

\section{Authors' contributions}

AM and CF made the ECD and ETD measurements and wrote the article. MJS created the bioinformatics tools and wrote the article. SH conceived the project and wrote the review article. All authors read and approved the final manuscript.

\section{Acknowledgements}

The Proteome Exploration Laboratory is supported by the Gordon and Betty Moore Foundation through grant GBMF775 and the Beckman Institute; CF is supported by Fulbright, PROLAB (ASBMB) through a Wood-Whelan fellowship from IUBMB and from Fundação para a Ciência e Tecnologia (Lisbon, Portugal) with both a project grant (PTDC/MAR-BIO/2174/2012) and a post-doctoral fellowship (SFRH/BPD/79271/2011).

\section{Author details}

${ }^{1}$ Proteome Exploration Laboratory, Beckman Institute, California Institute of Technology, BI 211, MC 139-74, Pasadena, CA 91125, USA. ${ }^{2}$ Instituto de Tecnologia Química e Biológica, Universidade Nova de Lisboa, Av. da República, 2780-157 Oeiras, Portugal.

Received: 9 March 2015 Accepted: 19 May 2015

Published online: 21 June 2015

\section{References}

Bonenfant D, Coulot M, Towbin H, Schindler P, van Oostrum J (2006) Characterization of histone $\mathrm{H} 2 \mathrm{~A}$ and $\mathrm{H} 2 \mathrm{~B}$ variants and their post-translational modifications by mass spectrometry. Mol Cell Proteomics 5(3):541-552. doi:10.1074/mcp.M500288-MCP200

Cannon J, Lohnes K, Wynne C, Wang Y, Edwards N, Fenselau C (2010) Highthroughput middle-down analysis using an Orbitrap. J Proteome Res 9(8):3886-3890. doi:10.1021/pr1000994

Carvalho PC, Xu T, Han X, Cociorva D, Barbosa VC, Yates JR 3rd (2009) YADA: a tool for taking the most out of high-resolution spectra. Bioinformatics 25(20):2734-2736. doi:10.1093/bioinformatics/btp489

da Cunha JP, Nakayasu ES, de Almeida IC, Schenkman S (2006) Post-translational modifications of Trypanosoma cruzi histone H4. Mol Biochem Parasitol 150(2):268-277. doi:10.1016/j.molbiopara.2006.08.012

French WR, Zimmerman L, Schilling B, Gibson BW, Miller CA, Townsend RR, Sherrod SD, Goodwin CR, McLean JA, Tabb DL (2014) Wavelet-based peak detection and a new charge inference procedure for MS/MS implemented in ProteoWizard's msConvert. J Proteome Res. doi:10.1021/pr500886y

Garcia BA (2010) What does the future hold for top down mass spectrometry? J Am Soc Mass Spectrom 21(2):193-202. doi:10.1016/j.jasms.2009.10.014

Garcia BA, Shabanowitz J, Hunt DF (2007) Characterization of histones and their post-translational modifications by mass spectrometry. Curr Opin Chem Biol 11(1):66-73. doi:10.1016/j.cbpa.2006.11.022

Guedes S, Vitorino R, Domingues MR, Amado F, Domingues P (2011) Glycation and oxidation of histones $\mathrm{H} 2 \mathrm{~B}$ and $\mathrm{H} 1$ : in vitro study and characterization by mass spectrometry. Anal Bioanal Chem 399(10):3529-3539. doi:10.1007/ s00216-011-4679-y

Jung HR, Sidoli S, Haldbo S, Sprenger RR, Schwammle V, Pasini D, Helin K, Jensen ON (2013) Precision mapping of coexisting modifications in histone $\mathrm{H} 3$ tails from embryonic stem cells by ETD-MS/MS. Anal Chem 85(17):8232-8239. doi:10.1021/ac401299w

Kalli A, Hess S (2012) Electron capture dissociation of hydrogen-deficient peptide radical cations. J Am Soc Mass Spectrom:1-12

Kalli A, Sweredoski MJ, Hess S (2013) Data-dependent middle-down nano-liquid chromatography-electron capture dissociation-tandem mass spectrometry: an application for the analysis of unfractionated histones. Anal Chem 85:3501-3507

Kawasaki H, Isaacson T, Iwamuro S, Conlon JM (2003) A protein with antimicrobial activity in the skin of Schlegel's green tree frog Rhacophorus schlegelii (Rhacophoridae) identified as histone H2B. Biochem Biophys Res Commun 312(4):1082-1086

Liu X, Inbar Y, Dorrestein PC, Wynne C, Edwards N, Souda P, Whitelegge JP, Bafna V, Pevzner PA (2010) Deconvolution and database search of complex tandem mass spectra of intact proteins: a combinatorial approach. Mol Cell Proteomics 9(12):2772-2782. doi:10.1074/mcp.M110.002766

Lu S, Xie YM, Li X, Luo J, Shi XQ, Hong X, Pan YH, Ma X (2009) Mass spectrometry analysis of dynamic post-translational modifications of $\mathrm{TH} 2 \mathrm{~B}$ during spermatogenesis. Mol Hum Reprod 15(6):373-378. doi:10.1093/molehr/gap028

MacLean B, Tomazela DM, Shulman N, Chambers M, Finney GL, Frewen B, Kern R, Tabb DL, Liebler DC, MacCoss MJ (2010) Skyline: an open source document editor for creating and analyzing targeted proteomics experiments. Bioinformatics 26(7):966-968. doi:10.1093/bioinformatics/btq054

Moradian A, Kalli A, Sweredoski MJ, Hess S (2014) The top-down, middle-down, and bottom-up mass spectrometry approaches for characterization of 
histone variants and their post-translational modifications. Proteomics 14(4-5):489-497. doi:10.1002/pmic.201300256

Pesavento JJ, Mizzen CA, Kelleher NL (2006) Quantitative analysis of modified proteins and their positional isomers by tandem mass spectrometry: human histone H4. Anal Chem 78(13):4271-4280. doi:10.1021/ac0600050

Phanstiel D, Brumbaugh J, Berggren WT, Conard K, Feng X, Levenstein ME, McAlister GC, Thomson JA, Coon JJ (2008) Mass spectrometry identifies and quantifies 74 unique histone $\mathrm{H} 4$ isoforms in differentiating human embryonic stem cells. Proc Natl Acad Sci U S A 105(11):4093-4098. doi:10.1073/ pnas.0710515105

Schilling B, Rardin MJ, MacLean BX, Zawadzka AM, Frewen BE, Cusack MP, Sorensen DJ, Bereman MS, Jing E, Wu CC, Verdin E, Kahn CR, Maccoss MJ, Gibson BW (2012) Platform-independent and label-free quantitation of proteomic data using MS1 extracted ion chromatograms in skyline: application to protein acetylation and phosphorylation. Mol Cell Proteomics 11(5):202-214. doi:10.1074/mcp.M112.017707

Shechter D, Dormann HL, Allis CD, Hake SB (2007) Extraction, purification and analysis of histones. Nat Protoc 2(6):1445-1457. doi:10.1038/nprot.2007.202

Wu SL, Kim J, Hancock WS, Karger B (2005) Extended Range Proteomic Analysis (ERPA): a new and sensitive LC-MS platform for high sequence coverage of complex proteins with extensive post-translational modifications-comprehensive analysis of beta-casein and epidermal growth factor receptor (EGFR). J Proteome Res 4(4):1155-1170. doi:10.1021/pr050113n

Young NL, DiMaggio PA, Plazas-Mayorca MD, Baliban RC, Floudas CA, Garcia BA (2009) High throughput characterization of combinatorial histone codes. Mol Cell Proteomics 8(10):2266-2284. doi:10.1074/mcp.M900238-MCP200

Young NL, DiMaggio PA, Garcia BA (2010) The significance, development and progress of high-throughput combinatorial histone code analysis. Cell Mol Life Sci 67(23):3983-4000. doi:10.1007/s00018-010-0475-7

\section{Submit your manuscript to a SpringerOpen ${ }^{\circ}$ journal and benefit from:}

- Convenient online submission

- Rigorous peer review

- Immediate publication on acceptance

Open access: articles freely available online

- High visibility within the field

- Retaining the copyright to your article

Submit your next manuscript at $>$ springeropen.com 